\title{
Análisis del viaje como uno de los ejes transformadores del proceso educativo
}

\section{Analysis of the trip as one of the transforming axes of the educational process}

Elevar la conciencia de las personas es el camino para humanizar el mundo ¡Viaja y Aprende! Óscar Pérez (Fundador de Pola Social).

María Abril-Sellarés

Profesora de la EUTDH-UAB y Coordinadora del máster en Turismo y Humanidades de la UAB maria.abril@uab.cat Código Orcid: https://orcid.org/0000-0003-4969-6304

María del C. Azpelicueta Criado Investigadora de la Universitat de les Illes Balears m.azpelicueta@uib.eu Código Orcid: https://orcid.org/0000-0002-8660-204X

María D. Sánchez-Fernández Profesora ayudante, doctor en la Universidade da Coruña maria.sanchezf@udc.es Código Orcid: https://orcid.org/0000-0001-9065-1699

Forma sugerida de citar: Abril-Sellarés, María, Azpelicueta Criado, María del Carmen, y SánchezFernández, María Dolores (2019). Análisis del viaje como uno de los ejes transformadores del proceso educativo. Universitas, 29, pp. 63-78. 


\title{
Resumen
}

La asociación del término "viaje" a vocablos como divertimento, erudición, periplo, ocio, vacación..., siendo añadido en estos últimos años a la entrada de conceptos tan importantes como interculturalidad o responsabilidad social y a nuevas formas de compartir aficiones, ideas y sensaciones, provocan que dicha locución de un salto cualitativo y amplié su significado, incluyéndola en el ámbito educativo (Aguerrondo, 2008).

La concepción amplia de turismo, entendida en nuestra Europa Occidental, nace a partir del periodo de la postguerra de la Segunda Guerra Mundial, acelerando su desarrollo a partir de los años sesenta (Salgueiro, 2002), si bien tenemos dataciones de que entre finales del siglo XVI y los siglos XVII-XVIII se produjo un planteamiento clave: el uso del viaje para formar a jóvenes universitarios adinerados de escuelas básicamente privadas, que durante uno o dos años, se embarcaban en la aventura mediterránea para conocer los orígenes de la cultura occidental europea, situados en Italia y Grecia. Este paradigma de viaje fue denominado "el Grand Tour" (Katharina-Lau, 2012).

El presente trabajo hace una aproximación a cómo el viaje puede ser uno de los ejes transformadores del proceso educativo, aplicando procesos de proactividad e interacción que permitan una mejor formación al estudiante para su posterior entrada al mundo laboral.

La metodología empleada es exploratoria con un análisis sistematizado de múltiples y variadas publicaciones científicas, que permitan sustentar un futuro constructo sobre el viaje educativo.

\section{Palabras clave}

Educación, viaje, turismo, profesional, aprendizaje, cocreación.

\begin{abstract}
The association of the term "trip" to words such as amusement, erudition, journey, leisure, vacation..., being added in recent years to the entry of concepts as important as interculturality or social responsibility and new ways of sharing hobbies, ideas and sensations, provoke that this locution of a qualitative leap and broadened its meaning, including in the educational field (Aguerrondo, 2008).

The broad conception of tourism, as understood in our Western Europe, is born from the postwar World War II, accelerating its development from the 1960s (Salgueiro, 2002), although we have dates that between the end of the century 16th and the 17th-18th centuries there was a key approach: the use of the trip to train wealthy university students of basically private schools, who for one or two years embarked on the Mediterranean adventure to learn about the origins of Western European culture, located in Italy and Greece. This travel paradigm was called "The Grand Tour" (Katharina-Lau, 2012).

The present work makes an approximation to how the trip can be one of the transforming axes of the educational process, applying processes of proactivity and interaction that allow a better formation to the student for his later entry to the working world.

The methodology used has an exploratory character with a systematized analysis of multiple and varied scientific publications, which allow to support a constructive future on the educational journey.
\end{abstract}

\section{Keywords}

Education, trip, tourism, professional, learning, crocreation. 


\section{Introducción}

En nuestra amplia trayectoria humana, muchas y variadas han sido las formas de desplazamiento del hombre, tanto a corta como a larga distancia. En el caso de la Europa Occidental, la historia evolutiva del propio viaje ha pasado por diferentes fases y concepciones (Moreno Garrido, 2012).

Si nos transportamos a la antigüedad clásica (griegos y romanos), los viajes no se produjeron, únicamente, por causas de supervivencia, comercio o poder, sino que existieron otras génesis que habilitaron el desplazamiento por un período de tiempo concreto, de grupos de personas con unas necesidades fijadas. De modo que, entre las principales motivaciones que podemos enumerar están: el ocio con los juegos olímpicos, la religión con las fiestas dedicadas a los dioses y finalmente el aprendizaje de nuevas teorías y materias tanto sociales como científicas, que impulsaron los viajes para conocer a los grandes del pensamiento humanístico y aprender de ellos. Fue, justamente, esta última razón la que permitió darle al viaje un nuevo sentido, entendiéndolo como un instrumento que permitía llegar a los lugares donde se impartía el conocimiento (Cloe-Sampaio, 2004). Si bien, en esos momentos, aún no se había alcanzado el caso de que fuese el propio viaje, el eje del proceso educativo.

En la Edad Media, los viajes basados en la fe se mantuvieron, así las peregrinaciones o las guerras santas fueron elementos claves en los flujos de desplazamiento de determinadas comunidades sociales o de individuos, en concreto. Por su parte, aquellos viajes motivados para aprender de los grandes maestros del pensamiento se vieron drásticamente reducidos, y sólo algunos privilegiados buscaron las enseñanzas más allá del espacio donde residían. Así una de las clases privilegiadas como fue la eclesiástica, tuvo la capacidad de viajar para aprender, si bien el viaje no se entendía en sí mismo como una herramienta para el aprendizaje, pero sí que sirvió como un instrumento para llegar a los lugares donde había la posibilidad de formarse, aunque fuese de modo restrictivo (Jafari, 1992), también encontramos la figura del compagnon, un aprendiz que para ejercer la profesión en los gremios de constructores (García-Vergara, \& Ortega-Sanz, 2011) tenía que recorrer diversos lugares estudiando las catedrales y otras construcciones relevantes, aspecto que conlleva el inicio de un viaje de formación para visitar diferentes ciudades.

Con el advenimiento de la etapa moderna, que podemos establecer entre principios del siglo XV a finales del siglo XVII, época que se considera como la de la Revolución Científica, podríamos suponer que la concepción 
del viaje como generadora de aprendizaje podría adquirir un nuevo significado - pero a pesar de la entrada de nuevas ideas, conocimientos y símbolos, se siguió vislumbrando al viaje como una forma de traslado para llegar a un destino, y no como un fin en sí mismo y, en este caso como un viaje educativo (Moreno Garrido, 2012).

Y la generación de una nueva concepción del mundo del viaje entrelazado con el mundo de la educación no se producirá hasta iniciado el siglo XX. El cambio se pertrecha en Gran Bretaña, donde la enseñanza pública comenzó a ganar popularidad y donde el aprendizaje grupal empezaba a ser considerado como un factor altamente positivo frente a la tutoría privada que había imperado hasta ese momento (Katharina-Lau, 2012). Ya no se trataba sólo de un planteamiento de cómo aprender sino de qué manera hacerlo. Así, y gracias a los descubrimientos de los conjuntos arqueológicos italianos de Pompeya y Hercolano (como principales centros), estudiar en el extranjero junto a otros compañeros empezó a adquirir una fuerza que en períodos anteriores no había tenido y donde el propio viaje también generaba aprendizaje (Jafari, 1992). Este viaje fue conocido como un viaje iniciático, llamándolo el Grand Tour (Salgueiro, 2002) y se convirtió en una forma activa y experiencial del conocimiento.

El Grand Tour, por tanto, se puede entender como un viaje juvenil, iniciático que se extenderá a lo largo del tiempo a ámbitos distintos del academicista y donde el propio viaje formará parte del aprendizaje para este segmento poblacional (los jóvenes), siendo considerado - además - por algunos autores, como Suárez Huerta un viaje iniciático para adquirir "un bagaje cultural fundamental para su desarrollo personal y profesional" (Suárez-Huerta, 2011, p. 256). En este momento, exactamente es cuando la idea del viaje como elemento formativo empezará a no parecer extraño y hasta llegará a convertirse, para ciertas clases sociales, en algo habitual.

Si inicialmente el Grand Tour incluía, parte de Francia, París, Italia, Florencia, Roma, Nápoles y parte de Suiza, a partir del siglo XIX, un nuevo país entrará a formar parte de este concepto de viaje educativo: Grecia, y con ella, se completaba el abanico humanístico que configuraba a los clásicos.

En definitiva, este producto turístico; denominémosle así con estos términos, puesto que incluía diversos elementos que, en la actualidad entendemos como oferta turística, tales como el alojamiento, el transporte, los recursos patrimoniales, y algunos servicios complementarios como el de los guías; pretendía que los jóvenes estudiantes obtuviesen una experiencia vivencial vinculada a su continua formación académica, a lo largo de dos años 
y con un tutor que no solo era su maestro sino también su guía, de este modo estamos frente a la realización de un viaje con un aprendizaje adaptado a las necesidades de los pupilos (López-Martínez, 2015).

La eclosión en la Revolución Industrial de las máquinas de vapor, tanto por tierra como por mar, la construcción de las nuevas infraestructuras viarias, las exposiciones universales, la exploración de nuevos territorios, entre otros elementos, hicieron germinar una nueva perspectiva del concepto del viaje (Barrow, 2008). Primero, se hacía extensivo a otras clases sociales para las que - hasta aquel momento - era impensable poder viajar, y segundo, dejaba de tener, el viaje, aquel concepto educativo para las clases altas yendo a buscar un nuevo sentido, básicamente de ocio y entretenimiento. Así, como la consideración de la industria del turismo como una actividad económica a partir de 1911. (Scutariu, 2009).

En pleno siglo XX y -más concretamente entre las décadas de los 50 y de los 80 - el turismo tuvo una característica muy alejada de lo que se podría entender como viaje educativo, dado que las motivaciones de la demanda eran básicamente las de ocio y entretenimiento, apareciendo aquello que se conoce como el turismo de masas, aunque sea un término, tal y como señala Bertram, "problemático" (Bertram, 2002).

Viajar, por tanto, forma parte de nuestro bagaje personal y de nuestro mundo (Salgueiro, 2002) y permite ampliar horizontes, en un sentido claro de aprendizaje, si del viajero subyace un carácter descubridor, observador, pensador o reflexor será la señal, en la cual el viaje está logrando esa parte no sólo lúdica sino cognitiva. No hay que olvidar, por otro lado que, tal y como lo contempla la OMT en su artículo 7.2 el turismo, donde el elemento inherente y primordial va a ser el viaje como eje experiencial, memorable (Coelho, Gosling y Almeida, 2018), que no sólo satisface ciertas necesidades sino que ha pasado a formar parte de los derechos que tienen los ciudadanos (UNWTO, 1999), convirtiéndose en un fenómeno importante para los países a niveles económicos, culturales y sociales, donde un segmento de su sector terciario o cuaternario explota los elementos centrales de la producción y el consumo.

\section{El viaje educativo}

Cuando hablamos de turismo, el eje será el viaje y éste es el que nos permite romper con la rutina diaria, descubrir nuevos lugares, conocer gente, 
abrir nuestra mente, estimular nuestros sentidos y en algunos casos conseguir que sea una vía de aprendizaje más (Barrow, 2008) a la que añadir a los modelos tradicionales y a los modelos virtuales, lo que no siempre resulta ni fácil ni evidente (Ferrés, 2008). Podemos viajar solos o acompañados; en alta, media o baja temporada; cerca o lejos de casa; por motivos totalmente dispares como disfrutar del "dark tourism" (Séraphin, 2017); el sol y playa; el viaje extremo o el de aventura, entre otros. Pero, en definitiva, el viaje nos permite durante unos días escapar de la realidad más cercana y evadirnos o ensoñarnos con elementos que marcan las distintas motivaciones viajeras. Pero ¿y si el viaje no fuese una vía de escape sino una herramienta de aprendizaje tal y como había sido en épocas anteriores y dispares? Muy probablemente nos daría la capacidad de probar un conjunto de experiencias que no nos hubiésemos planteado, posiblemente, al inicio de este (Sperano, Roberge, Bénech, Trgalova, \& Andruchow, 2019).

La primera condición para que el viaje sea un viaje educativo es que el viajero tenga una actitud positiva, una mente abierta y que acepte que el viaje le reportará conocimiento, por lo que es conveniente que antes de iniciar un viaje educativo haya una planificación adecuada y análoga (García-Vergara, \& Ortega-Sanz, 2011). La segunda condición es que haya una voluntad clara de querer mejorar, de nada sirve un viaje educativo, si el viajero que lo va a realizar cree y piensa que su aprendizaje, ya ha cerrado el ciclo correspondiente. Así tenemos que la idea de experiencia se ve envuelta de un cierto pragmatismo donde necesariamente existe una relación entre poder hacer, experimentar y aprender. En otras palabras, se aprende mientras se hace el viaje (Larrosa, 2011).

Con todas las premisas, anteriormente mencionadas es necesario plantearse el viaje desde dos perspectivas que a priori pueden parecer muy distintas, pero que tienen elementos que son comunes (Mandelli, \& La Rocca, 2014). En el objetivo que nos ocupa culturizar, educar y viajar son tres verbos que conforman los fundamentos del viaje como herramienta para el cambio en el proceso educativo. De modo que no podemos desgajar uno de ellos sin romper el frágil equilibrio que mantienen, ya que estamos estableciendo que el viaje va a ser nuestro eje transformador. Las perspectivas analizadas en este artículo son: la perspectiva turística y la perspectiva educativa. 


\section{La perspectiva turística}

El viaje educativo se encontraría en una fase considerada larvaria, tal y como hemos comprobado, si bien hay un conjunto de sub-tipologías turísticas que recogen partes educativas, en la mayoría de los casos, el viaje continúa siendo un instrumento para partir y alcanzar un punto determinado, mientras que realizamos en un espacio cerrado, un aprendizaje (García-Vergara, \& Ortega-Sanz, 2011). De hecho, lo que se traslada es un concepto de espacio físico donde se imparten una serie de horas de docencia establecidas, aunque consideramos que en los viajes educativos habría un valor añadido, que es el aprovechamiento que pueden hacer las personas que demandan este producto a nivel turístico, puesto que es fehaciente el traslado al lugar elegido, la realización de dispendios y el esbozo de una experiencia con un producto, un sistema o un servicio, a través de los mapas de viaje (Kalbach, 2016).

Pero, evidentemente, el viaje educativo se puede analizar desde un prisma de producción, siendo sus resultados, los siguientes:

- Algunos autores, como Pawlowska, Martínez-Roget y Pereira-López, hablan de turismo académico para describir aquellos viajes que los estudiantes de unas universidades concretas realizan a otras universidades con el objetivo de seguir su aprendizaje en otro centro educativo con una perspectiva sociocultural distinta a la que poseen en su país de origen. En el caso europeo estos viajes académicos vienen en su mayoría avalados por la Unión Europea (UE), a través de distintos programas siendo el más conocido el programa Erasmus - que fue galardonado hace unos años con el Premio Príncipe de Asturias como reconocimiento a su labor por su desarrollo de Europa-, en el que pueden acceder a él, tanto estudiantes como profesores. En este caso la perspectiva del viaje es finalista, y no durante el viaje ni en el viaje en sí mismo considerado (Pawlowska, 2011), (Martínez-Roget, Pereira-López, \& Pawlowska, 2013).

- Por su parte, autores como Marrero-Rodríguez, Abdul-Jalbar, Orduna y Urpí señalan que el turismo cultural puede ser entendido como turismo educativo por la propia motivación de la demanda turística, así como por los recursos patrimoniales a los que acceden, aunque sea contemporáneamente negocio, ocio y aprendizaje (Marrero-Rodríguez, \& Abdul-Jalbar, 2012). Con lo que, en este caso, la activi- 
dad lúdica que es el viaje se convierte en una actividad que permite el crecimiento de cada uno de los viajeros y, que ayuda a forjar su personalidad a través de las experiencias que va obteniendo en dicho viaje (Orduna, \& Urpí, 2010).

- Un trabajo de investigadores de universidades rusas nos remarca que hay una serie de tecnologías educativas implementadas por el Dr. Shogan y la Dr. Storozhakova como un factor de desarrollo axiológico de la personalidad de los estudiantes en el contexto de la educación espiritual y moral en el ámbito del turismo (Frolova, Polenova, Khoruzhiy, \& Budaev, 2018), en el cual se presenta la experiencia de desarrollar el proyecto de "viaje contemplativo a la ciudad de San Petersburgo", aplicando los susodichos métodos en las actividades de excursión.

- Si buscamos la especialización en el turismo educativo, encontramos aquello que se conoce como turismo idiomático, una tipología que muestra una doble forma de proceder, por un lado, aprender una lengua mientras se está haciendo turismo en una ciudad, casos de estudiantes que en su viaje de fin de curso o de paso por un territorio concreto deciden aprovechar todas las actividades culturales para practicar y seguir aprendiendo una lengua, o aquellas personas que vienen a nuestro país para aprender el español como lengua extranjera y que al mismo tiempo aprovechan para conocer los recursos turísticos de la zona. Pero su aprendizaje se encuentra en un espacio escolástico concreto y no en el hecho de la visita, propiamente dicha. Una de las ofertas más conocidas para este segunda manera de proceder son los cursos de español como Lengua Extranjera, conocidos como ELE, siendo ésta una de las formas de demanda más habitual con una oferta que va más allá de los 600 centros educativos en toda España (Piédrola-Ortiz, \& Artacho-Ruiz, 2011).

\section{La perspectiva educativa}

Uno de los autores que más ha investigado la relación cultura y pedagogía es Trilla, el cual señala como los espacios culturales pueden ser educativos y viceversa: 
Todas las instituciones llamadas educativas son, por el mismo hecho de serlo, instituciones culturales; y lo mismo a la inversa. Si acaso, la diferencia entre unas y otras es sólo una diferencia de énfasis: las instituciones educativas ponen el acento en la transmisión o adquisición de la cultura, mientras las denominadas culturales lo ponen en la conservación (material), creación y uso de la cultura. Pero eso sólo es una diferencia de énfasis, ya que en las instituciones educativas además de la transmisión también hay elaboración y uso de la cultura. Y, por el otro lado, tampoco nadie negará que en las instituciones culturales existe también transmisión y aprendizaje. En definitiva, las unas se fijan más en unos determinados momentos del proceso cultural y las otras en otros, pero el objeto con el que ambas trabajan es justamente el mismo (Trilla, 2000, pp. 135-136).

En las nuevas tendencias de metodologías de aprendizaje en el ámbito europeo se han introducido - en estos últimos veinte años - nuevas formas, así como nuevos intentos de aproximación a las necesidades que tanto el mundo profesional como el mundo educativo requieren. La crisis de los modelos tradicionales de enseñanza pone en evidencia la celeridad de los cambios en un mundo que cada vez es más global y que tal y como señala Aguerrondo "desde principios del siglo XX la ciencia comenzó a dejar de ser observación del mundo e información para pasar a ser creación del mundo" (2008, p. 2).

Como consecuencia de estos cambios, se produce una nueva formulación educativa, donde producir y concebir conocimiento se puede hacer no sólo dentro del aula sino fuera de la misma (García-Vergara, \& Ortega-Sanz, 2011). Así se recupera, en cierto modo, el paradigma del Grand Tour, donde el turismo puede ayudar al viajero, sea o no estudiante, a desarrollar nuevas formas no sólo de aprendizaje sino de adquisición de competencias que permitan un acceso más fácil al mundo laboral. Evidentemente se aboga por un viaje educativo físico (Horey, Nicolacopoulos, Kashima, \& Mathisen, 2018), es decir, donde sea necesario el traslado del sujeto y no sólo para estar ahí, sino para implicarle de manera activa, a través de acciones proactivas y con un contacto directo con los agentes implicados (López-Martínez, 2015).

La facilidad con la que hoy se puede viajar, tanto de manera física como virtual, ha revolucionado al ser humano y con ello, los aspectos formativos que le acompañan de un modo total o parcial a lo largo de los distintos procesos por los que pasa su ciclo de vida. De modo que, plantearse unas posibles futuras aulas en este siglo XXI que permitan no sólo un conocimiento teórico sino práctico puede ser una idea potencialmente realizable, a través 
de - por un lado - todas las herramientas que internet nos permite, $\mathrm{y}$ - por otro lado - los distintos destinos turísticos, efectuando el viaje a los mismos, entendidos de un modo global y sin fisuras. En otras palabras, que el viaje sea parte del proceso educativo, que se entienda como la herramienta de aprendizaje y no como un mero vehículo que, simplemente, te traslada a un destino turístico (Henao-Álvarez, 1993).

Uno de estos casos se plasma en la comunicación presentada en UNIVEST11 por las profesoras Marisa García y Yolanda Ortega de la Universidad de Girona, en la que durante el curso 2010-2011 enmarcan la asignatura "Arquitecturas contemporáneas" (optativa) ligada a una experiencia fuera del aula, que es un viaje de estudios a la ciudad de Berlín, recuperando el viaje como actividad facilitadora del aprendizaje de una forma cooperativa, a través de toda la planificación y realización del propio viaje combinado con el estudio de la ciudad y un análisis seleccionador de sus obras arquitectónicas, estas últimas actividades realizadas en el aula. Esta experiencia plantea una docencia basada en la construcción activa del conocimiento; una autogestión en la forma del aprendizaje por parte de los estudiantes y entre los estudiantes, ya que hay que conseguir objetivos compartidos y realizar trabajo en equipo; "teniendo en cuenta que estas experiencias educativas representan un tipo de actividad pedagógica altamente formativa para los estudiantes", recomendando crear redes para la formación creativa y cooperativa universitaria y realizar actividades basadas en la búsqueda, creación y uso de esta herramientas y recursos (García-Vergara, \& Ortega-Sanz, 2011).

Otro de los casos es el relato que nos hace Julen Iturbe-Ormaetxe sobre la experiencia maravillosa del viaje en solitario que realiza alrededor del mundo y plasma en tres libros, Salva Rodríguez - profesor de instituto y viajero - señalando que "el viaje es una enorme fuente de aprendizaje y estos tres libros con un buen material para aprender: De la gente, del mundo, de la humanidad" y sobre la experiencia que se realiza por los docentes y los estudiantes en el grado de LEINN de la Universidad de Mondragón, en la que se utiliza "el viaje como uno de los principales estímulos de aprendizaje" (Iturbe-Ormaetxe Zamarripa, 2015).

Desde finales del siglo XX, las nuevas metodologías didácticas han permitido cambios y avances constantes, que nos permite afirmar que el alumno es, en pleno siglo XXI, un potencial sujeto activo de aprendizaje. Además, cuando se menciona el verbo enseñar se puede señalar que este implica tener experiencias y organizarlas para que el alumno haga las construcciones 
necesarias en sus modelos de formación. El viaje, por tanto, se puede convertir en una de las tantas vía de aprendizaje, donde se pueden desarrollar parte de las competencias que se exigen a los alumnos (Aguerrondo, 2008).

Por tanto, estrechar lazos entre los sistemas educativos y los viajes educativos o de carácter académico, podría convertirse en una necesidad que no por perentoria, no siempre podría ser de fácil resolución. Una de las posibilidades es que el viaje se pudiese fundamentar en un modelo basado en los componentes conectados, interactivos, sociales y ubicuos, poniendo especial énfasis en las expectativas y necesidades específicas de todos los agentes implicados, donde aptitudes y personalidad ya existentes permiten asumir o reforzar nuevas competencias. De este modo, se pretende que el estudiante deje de ser un sujeto pasivo para convertirse en un sujeto activo que busca en las distintas acciones que un viaje pueda permitir, pueda alcanzar la satisfacción de sus necesidades de aprendizaje y pueda dar a conocer las necesidades de los agentes implicados (Lévy-Leboyer, 2000). La parte más importante, es poder alcanzar una experiencia de carácter global como la que tiene el aprendizaje, donde cada uno de los elementos en uso sea capaz de reforzar la potencialidad de lo que venimos llamando un viaje educativo. Tal y como señala Cantero "la relación entre el turismo y la cooperación para el desarrollo está llamada a proponer nuevos escenarios en los cuales el turismo colabora con el objetivo de desarrollo humano en el mundo" (Cantero-Medina R., 2012).

Para ello, en un viaje educativo se le pedirá al viajero - aventurero, bohemio, errabundo, estudiante, excursionista, explorador, navegante, nómada, peregrino, trotamundos, turista, usuario, - de este, que tenga un pensamiento sistémico, donde la observación de los objetos se tendrá que realizar como si de fenómenos complejos se tratasen. Consecuentemente, a este tipo de viajes se tendrá que darles un nuevo enfoque que permita la posibilidad de ver una realidad compleja en sus interrelaciones y diversos elementos (Argudin, 2006). De modo que en la preparación de un viaje educativo no será suficiente con visitar determinados destinos, sino saber por qué se visitan, qué implican en el aprendizaje, si se buscan objetivos que permitan obtener las bases para un pensamiento crítico y si permiten un trabajo en equipo. En definitiva, un viaje educativo ha de tener la capacidad de desarrollar un conjunto de competencias dirigidas a unas mejoras tanto personales como profesionales.

En este momento es sugestivo, comentar el uso de aplicaciones basadas en la realidad interactiva y aumentada para presentar el concepto en insti- 
tuciones de educación superior desde un enfoque pedagógico y tecnológico con el objeto de preparar a los nuevos estudiantes para su viaje educativo (Nguyen, Muilu, Dirin, \& Alamäki, 2018) o la introducción de herramientas como por ejemplo los mapas de viaje, que son representaciones gráficas y textuales - instrumentos de visualización - que pretenden esbozar una experiencia en el tiempo con un producto, un sistema o un servicio (Kalbach, 2016), que nos servirá como método de diseño anticipado y colaborativo para apoyar la creación de actividades pedagógicas para ser usadas por los docentes y como método de planificación de proyectos prospectivos y retrospectivos para los académicos (Sperano, Roberge, Bénech, Trgalova, \& Andruchow, 2019) o la implantación de programas de desarrollo comunitario, metodología o viajes educativos para promulgar el concepto de la ciudadanía global en el desarrollo de los currículos de los estudiantes de educación superior (Horey, Nicolacopoulos, Kashima, \& Mathisen, 2018).

Finalmente, en la actualidad, hablar de competencias básicas y específicas que puedan situar a los estudiantes en la línea de su mejor desarrollo tanto profesional como personal no es ajeno a ninguno de ambos ámbitos (De Miguel-Díaz, 2006) y por ello, hay que aprovechar las oportunidades que un viaje les puede ofrecer para su completo crecimiento, entendiendo que el viaje puede ser una de las herramientas integrantes de las consideradas nuevas pedagogías y, por ende, para presente y para futuro, uno de los ejes transformadores del proceso educativo.

\section{Conclusiones, reflexiones y retos}

El hombre intrínsecamente hablando, desde siempre, ha sentido y siente la necesidad de sociabilizar y de viajar, evidentemente las motivaciones han variado en el tiempo. Muchas son la formas de entender y nombrar tipologías de turismo, si bien en este estudio nos hemos centrado en el turismo educativo, el cual ha sido planteado como un nuevo reto, donde se pueden llegar a rescatar ciertas ideas del pasado para poderlas aplicar con todas las tecnologías en un presente, donde la globalización nos puede hacer perder ciertos aspectos de las distintas idiosincrasias que con un viaje se pretenden conocer. Por ello, en este estudio hemos intentado iniciar una reflexión desde las dos perspectivas que unen a la investigación: el viaje y la educación, pero a la vez, somos conscientes de que quedan muchas cuestiones sin re- 
solver y aún nos podríamos atrever a decir, incluso sin explorar. No obstante, se pueden extraer algunas conclusiones preliminares que pueden ayudar en un futuro a seguir esta investigación. Entre las conclusiones destacamos:

- La dispersión existente en el tema objeto de esta investigación, sin que de momento haya un análisis en profundidad que pueda aportar una nueva conceptualización y metodología y sea incluido tanto en el ámbito turístico como en el educativo.

- La dificultad de proporcionar pautas y productos turístico - educativos adecuados a la realidad de cada uno de los viajes que pueden adoptar la concepción de viajes educativos.

- La necesidad de entender que, en un proceso de aprendizaje, el viaje puede abrir nuevos retos para el desarrollo de determinadas competencias como el sentido crítico, el trabajo en equipo o el conocimiento adquirido.

- La incógnita sobre las formas de mostrar la idea de que un viaje turístico pueda ser una herramienta que puede aportar un valor añadido al mundo educativo, si el turismo se ve cada día más como un sector economicista y no como un sector humanista, donde en ocasiones se fomenta más el consumismo y menos su carácter cultural, patrimonial y social.

- La recuperación de los significados de los viajes educativos de antaño desde el turismo y las nuevas metodologías educativas, en los que sus contenidos se actualizan, es decir, en parte hay una co-creación e implicación del estudiante en la propia materia, no sólo para ser atractivos sino para que tengan una aportación en la propia educación de los viajeros-estudiantes, sin que por ello tengan la consciencia de que es sólo un adoctrinamiento, ya que son participantes y creadores de la magnitud de conocimiento, que es trasladado en infinidad de contextos y espacios de vivencia.

- La búsqueda de herramientas con la pretensión de que todos los agentes implicados se planteen el concepto de viaje educativo desde la esencia fundamental del mismo, y, no únicamente como instrumento útil de traslado y de llegada a un destino para realizar in situ un proceso académico, dejando de lado el propio viaje que ha llevado a ese sitio al viajero.

- La necesidad de potenciar un aprendizaje en movilidad, es decir, que la adquisición de conocimiento puede y debe de ocurrir en cualquier lugar, gracias a la incidencia que tiene en nuestro mundo, la 
denominada educación expandida, en la que se debería usar entre otros elementos axiales y transformadores, el concepto de viaje, en su amplia significancia.

\section{Bibliografía}

Aguerrondo, I. (2008). Conocimiento complejo y competencias educativas. UNESCO-IBE. Ginebra: UNESCO-IBE. Recuperado de https://goo.gl/ jL99nW (24-10- 2018)

Argudin, Y. (2006). Educación basada en competencias. Nociones y antecedentes. México DF: Trillas.

Barrow, S. (2008). Edutourism, edutourism, edutourism. There is no darkness but ignorance. Tourism Society Journal (137), 2-3.

Bertram, M. (2002). El turismo de masas: un concepto problemático en la historia del siglo XX. Historia Contemporánea, 125-156.

Cantero-Medina, R. (2012). Turismo responsable, cooperación internacional y educación para el desarrollo. En M. Rivera-Mateos y L. Rodríguez García, Turismo responsable, sostenibilidad y desarrollo local comunitario (pp. 323-345). Córdoba: Cátedra Intercultural.

Cloe-Sampaio, C. (2004). El turismo como fenómeno histórico. Estudios y perspectivas en turismo, 13, 209-302.

Coelho, M., Gosling, M., \& Almeida, A. (2018). Tourism experiences: Core processes of memorable trips. Journal of Hospitality and Tourism Management (37), 11-22. https://doi.org/10.1016/j.jhtm.2018.08.004

De Miguel-Díaz, M. (2006). Metodologías para optimizar el aprendizaje. Segundo objetivo del Espacio Europeo de Educación Superior. Revista Interuniversitaria de Formación del profesorado, 20(3), 71-91.

Ferrés, J. (2008). La educación como industria del deseo: Un nuevo estilo comunicativo. Barcelona: Gedisa S.A.

Frolova, I., Polenova, A., Khoruzhiy, L. Budaev, P., \& Storozhakova, E. V. (2018). Educational technologies as a factor of axiological development of students' personality in sphere of tourism. Financial and Economic Tools Used in the World in the Hospitality Industry-Proceedings. Lumban Gaol, Filimonova \& Maslenikov Eds. London: Taylor \& Francis Group. García-Vergara, M., \& Ortega-Sanz, Y. (2011). El viaje como experiencia de aprendizaje. (Univest11, Ed.) $U d G$. Recuperado de https://goo.gl/3V9PvQ (411-2018). 
Henao-Álvarez, O. (1993). El aula escolar del futuro. Revista Educación y Pedagogía $(8$ y 9$)$.

Horey, D. T., Nicolacopoulos, T., Kashima, E., \& Mathisen, B. (2018). Global Citizenship and Higher Education: A Scoping Review of the Empirical Evidence. Journal of Studies in International Education, 22(5), 472-492. doi:10.1177/1028315318786443

Iturbe-Ormaetxe Zamarripa, J. (2015). El viaje en solitario como experiencia de aprendizaje: Salva Rodríguez. Consultoría Artesana en Red, 1-2. Recuperado de https://goo.gl/5GjKL4 (17-10-2018).

Jafari, J. (1992). Significado sociocultural y educacional del turismo de juventud. Papers de Turisme (8-9), 39-46.

Kalbach, J. (2016). Mapping experiences: A complete guide to creating value though journeys, blueprints and diagrams. Sebastopol: O'Reilly Media, Inc.

Katharina-Lau, M. (2012). El grand tour: el signo de educación de un gentleman. De Arte, 11, 131-142.

Larrosa, J. (21 de agosto de 2011). Conversando sobre escola, experiência e formaçao docente (S. Fernandes, y M. Silveira-Barbosa, Entrevistadores). Recuperado de https://goo.gl/Y9QzNx (11-10-2018).

Lévy-Leboyer, C. (2000). Gestión de las Competencias. Barcelona: Gestión.

López-Martínez, G. (2015). El Grand Tour: Revisión de un viaje antropológico. Gran Tour: Revista de Investigaciones Turísticas (12), 106-120.

Mandelli, A., \& La Rocca, A. (2014). From service experiences to augmented service journeys: Digital technology and networks in consumer services. En E. Baglieri y U. Karmarkar, Managing Consumer Services: Factory or Theater? Los Angeles: Springer International Publishing Switzerland. doi:10.1007/978-3-319-04289-3

Marrero-Rodríguez, R., \& Abdul-Jalbar, B. (2012). Turismo cultural y planificación del viaje: un estudio de caso. Tourism \& Magament Studies (8), 41-47. Recuperado de https://goo.gl/Zpj6Pt (14-10-2018).

Martínez-Roget, F., Pereira-López, X., \& Pawlowska, E. (2013). El turismo académico en Galicia: Otra forma de contribución de las universidades a las economías locales. Cuadernos de Turismo, 229-242.

Moreno Garrido, A. (2012). Historia del turismo: una investigación necesaria. Turismo y sostenibilidad: V Jornadas de Investigación en Turismo (pp. 105-126). Sevilla: Facultad de Turismo.

Nguyen, N., Muilu, T., Dirin, A., \& Alamäki, A. (2018). An interactive and augmented learning concept for orientation week in higher education. Inter- 
national Journal of Educational Technology in Higher Education, 1-15. doi:10.1186/s41239-018-0118-x

Orduna, G., \& Urpí, C. (2010). Turismo cultural como experiencia educativa de ocio. Polis. Revista Latinoamericana. Recuperado de https://goo.gl/ mTaCwV (10- 2018).

Pawlowska, E. (11 de agosto de 2011). El turismo académico: un análisis económico para el caso de Galicia. Tesis Doctoral. Santiago de Compostela, España: Universidade de Santiago de Compostela.

Piédrola-Ortiz, I., \& Artacho-Ruiz, C. (2011). El turismo idiomático: una oportunidad de desarrollo local para Córdoba. Revista de Análisis Turístico, 12, 49-61. doi: https://doi.org/10.1234/RAT2011n11

Salgueiro, V. (2002). Grand Tour; uma contribuiçao à historia do viajar por prazser e por amor à cultura. Revista Brasileira de História, 22(44). doi: http:// dx.doi.org/10.1590/S0102-01882002000200003

Scutariu, A. (2009). Tourism: Economic growth factor and essential element in regional development of Romania. Thesis. Roma, Italy.

Séraphin, H. (2017). Assessing dark tourism as a sustainable economic activity for emerging destinations using a multi criteria approach. En B. Korstanje M. y Handayani, Gazing at Death: Dark Tourism as an Emergent Horizon of Research (pp. 57-74). Nova Science-Publishers, Inc.

Sperano, I., Roberge, J., Bénech, P., Trgalova, J., \& Andruchow, R. (2019). Exploring new usages of journey maps: Introducing the pedagogical and the project planning journey maps. Advances in Intelligent Systems and Computing. 824-964-98220th. doi:10.1007/978-3-319-96071-5_99

Suárez-Huerta, A. (2011). El Grand Tour: un viaje emprendido con la mirada de Ulises. Isimu: Revista sobre Oriente próximo y Egipto en la antigüedad, 253-279.

Trilla, J. (2000). La cultura y sus mediaciones pedagógicas. En J.L. García Garrido (Ed.), La sociedad educadora, 215-244.

UNWTO (1999). UNWTO Organización Mundial del Turismo. Recuperado de Código Ético Mundial para el Turismo: https://goo.gl/9zCNyf (21-10-2018).

Fecha de recepción: 2018/11/18; Fecha de aceptación: 2019-02-02;

Fecha de publicación: 2019-03-01 